\title{
Circadian regulation of proprotein convertase subtilisin kexin type 9(PCSK9) for possible chronotherapy with PCSK9 inhibiters
}

\begin{abstract}
Cardiovascular diseases (CVDs) have become a public health problem despite increased availability of statins for decreasing hypercholesterolemia. Recently, pro protein Convertase subtilis in kexin 9(PCSK9) protein has been demonstrated to predispose inflammation, endothelial dysfunction and hypertension apart from its effects on low density lipoprotein (LDL)-receptor activity. Apart from causing increased in LDL cholesterol, PCSK9 has physio-pathological roles in several cardiovascular cells and more research is required on the regulation of PCSK9, its extra-hepatic activities focusing on these cells. The normal mean concentration of plasma PCSK9 is approximately $90 \mathrm{ng} / \mathrm{ml}$ which may increase to $100 \mathrm{ng} / \mathrm{ml}$ in hypercholesterolemia and $130 \mathrm{ng} / \mathrm{ml}$ in subjects with familial hypercholesterolemia. In a meta-analysis including 13,761 subjects, the relative risk for CVD was 1.25 (95\% CI: $1.14-1.38, \mathrm{P}<.001, \mathrm{I}=25 \%$ ) while compared highest to lowest level of PCSK9. It is clear that high circulating levels of PCSK9 may be associated with significantly increased risk of CVDs. These findings confirm for the first time that there is a nonlinear dose-response association between circulating PCSK9 level and risk of CVDs. The ODYSSEY OUTCOMES trial comprising of 18924 patients of acute coronary syndrome (ACS) showed that among patients with baseline LDL cholesterol $\geq 100 \mathrm{mg} / \mathrm{dL}$, alirocumab reduced MACE by $24 \%$ and all-cause death by $29 \%$ compared with placebo. However, despite these benefits, this trial provides a proof that alirocumab did not cause a significant decline in CAD and CV mortality, hence new strategies such as chronotherapy with PCSK9 inhibitors are needed to enhance efficacy of monoclonal antibodies, among these patients.
\end{abstract}

Keywords: statins, LDL cholesterol, inflammation, monoclonal antibody
Volume 7 Issue 5 - 2018

\author{
Jan Fedacko,' Ram B Singh, ${ }^{2}$ Viliam Mojto, ${ }^{3}$ \\ Dominik Pella, ${ }^{4}$ Galal Elkilany, ${ }^{5}$ Krasimira \\ Hristova, ${ }^{6}$ Daniel Pella' \\ 'PJ Safaric University, Kosice, Slovakia \\ ${ }^{2} \mathrm{H}$ alberg Hospital and research Institute, Moradabad \\ ${ }^{3}$ Comenius University, Slovakia \\ ${ }^{4}$ East Slovak Institute of Cardiovascular sciences, Slovakia \\ ${ }^{5}$ Gulf Medical College, UAE \\ ${ }^{6}$ National heart Hospital, Bulgaria
}

Correspondence: Jan Fedacko, MD, PhD, PJ Safaric University, Kosice, Slovakia, Tel: 42 I 91।315924,

Email janfedacko@hotmail.com

Ram B Singh, MD, FICN, FICC, Halberg Hospital and research Institute, Civil Lines, Moradabad, India, Tel: 919997794102,

Email rbs@tsimtsoum.net

Received: April 04, 2018 | Published: October 08, 2018

\section{Introduction}

Cardiovascular diseases (CVDs) have become a public health problem despite increased availability of drug therapy. Although there is an increased availability of low-cost statin therapy, a large unmet medical need remains for new and effective agents that are also well tolerated and safe, especially for patients unable to either tolerate statins or achieve optimal low density lipoprotein cholesterol (LDL-C) on all other current therapies. ${ }^{1}$ Recently, pro protein convertase subtilisin kexin 9 (PCSK9) protein has been demonstrated to predispose inflammation, endothelial dysfunction and hypertension apart from its effects on LDL-receptor activity. ${ }^{2-4}$ However, in a meta-analysis, circulating PCSK9 level as a continuous variable was not significantly associated with the risk of cardiovascular events indicating that larger, properly designed studies would be required to clarify the role of PCSK9 in cardiovascular risk. ${ }^{5}$ Clinical trials with PCSK9 inhibitors, including a meta-analysis published in 2017, have been conducted in which efficacy and safety of these agents, in reducing LDL-C up to $60 \%$ has been established. ${ }^{6-9}$ In preliminary trials, evolocumab and alirocumab, the leading PCSK9 inhibitors, have been demonstrated for their efficacy and safety in reducing cardiovascular events but alirocumab was associated with greater adverse effects than evolocumab., ${ }^{4,59}$ Poor nutrition during fetal life may cause under nutrition in new born with low birth weight which may be associated with increased susceptibility of these subjects to develop CVDs in later adult life. ${ }^{10}$ The increased susceptibility could be due to abnormal PCSK9 and lipoprotein metabolism which enhances the damage to concerned tissues that may increase CVD risk in later adult life. Further evidence indicate that PCSK9 has physiopathological dysfunctions in multiple cardiac and vascular cells and more research is required on the roles of PCSK9, its actions beyond hepatocytes but with a focus on these cells. In view of the potential benefits of PCSK9 inhibitors on reducing LDL cholesterol and CVDs, there is a need to develop new strategies to prevent the occurrence of adverse effects and increase the efficacy of these agents on longterm administration. ${ }^{11-13}$ Two of the new PCSK9 inhibitors directed against the binding site of LDL receptors in PCSK9 gene are approved in the European Union and the United States and several clinical trials targeted to PCSK9, are in way, but the benefits in mortality reduction are not very great. Further studies are necessary, to find out circadian concentrations of this molecule in the body, because circulating PCSK9 has a circadian rhythm which is synchronous with the synthesis of total and LDL cholesterol and is decreased by fasting in clinical studies. ${ }^{12}$ This view point is made to assess, if circadian rhythm of PCSK could be used for possible chronotherapy with PCSK9 inhibitors in the management of atherosclerosis.

\section{PCSK9 concentrations and risk of cardiovascular diseases}

There has been a controversy on the association of plasma PCSK9 levels and risk of CVDs. The circulation of PCSK9 is mainly in the 
blood plasma and an ELISA test was developed to measure total plasma PCSK9 concentration..$^{13}$ In a clinical study, plasma levels of PCSK9 were obtained among subjects with normal blood cholesterol $(n=254)$ and those with hypercholesterolemis $(\mathrm{n}=200)$ with and without treatment with statins or statin plus ezetimibe. In control subjects $(\mathrm{n}=254)$, the mean concentration PCSK $9(89.5 \pm 31.9 \mathrm{ng} / \mathrm{ml})$ in the plasma revealed significant positive correlation with age, fasting blood glucose, total and cholesterol and triglycerides. However, a new lossof-function R434W variant was found on sequencing of PCSK9 from subjects at the extremes of the normal PCSK9 distribution that was associated with lower levels of circulating PCSK9 and LDL-C. In subjects with high cholesterol, PCSK9 concentrations were greater than in control subjects $(99.3 \pm 31.7 \mathrm{vs} 89.5 \pm 31.9 \mathrm{ng} / \mathrm{ml}, P<0.04)$ and increase was consistent with drug therapy. The subjects with LDL receptor gene mutations known as familial hypercholesterolemia $(\mathrm{n}=139)$, treated with statins, showed greater PCSK9 levels compared to others $(147.01 \pm 42.5$ vs. $127.2 \pm 40.8 \mathrm{ng} / \mathrm{ml}, P<0.005)$, but decline in LDL cholesterol showed positive correlation consistent with plasma PCSK9 concentrations to a similar extent in both the subgroups. ${ }^{14}$

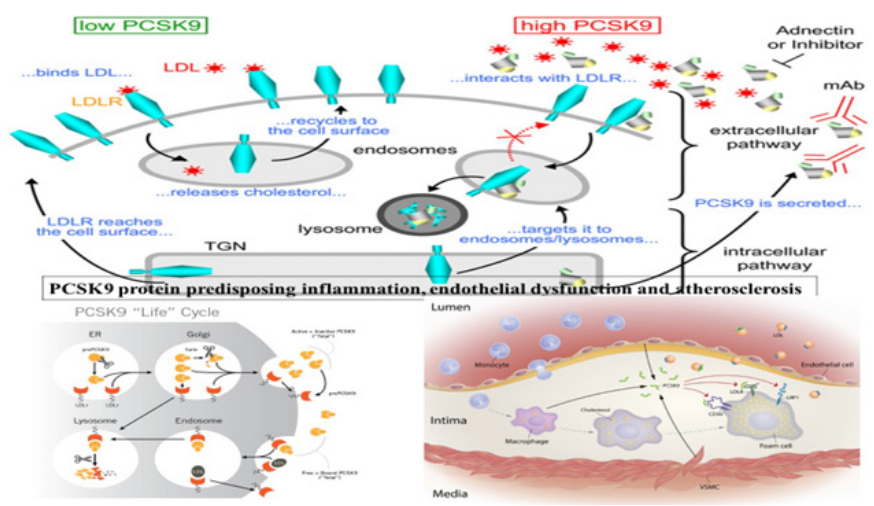

Figure I Effects of PCSK9 on endosome and lysosome and LDL-receptor as well as on progression of atherosclerosis by causing inflammation (modified from Google)

There is evidence that atherosclerosis is accelerated by increased levels of LDL-C which is under influence of PCSK9 through its capability to cause degradation of the LDL cholesterol receptor in the lysosome of the liver cells. ${ }^{15} \mathrm{~A}$ large number of recent advances made in recent years, in the understanding of PCSK9 physiopathology have revealed that the activity of PCSK9 is strongly regulated throughout by factors having definite influence on its release, transcription or by clearance and inactivation out of the cells. ${ }^{15}$ There is some evidence that LDL receptor activity and cholesterol synthesis as well as plasma PCSK9 levels are under influence of circadian alteration, dietary nutrients, fasting and exercise which may also influence plasma PCSK9 levels, which is secreted mainly by liver. ${ }^{12,13,16}$

In two recent meta-analysis, including 11 cohort studies comprising of 13,761 subjects, the relative risk for CVD was 1.25 (95\% CI: 1.14$1.38, \mathrm{P}<.001, \mathrm{I}=25 \%$ ) while compared highest to lowest level of PCSK9. ${ }^{4,5}$ It is clear that high circulating levels of PCSK9 may be associated with significantly increased risk of CVDs. These findings confirm for the first time that there is a nonlinear dose-response association between circulating PCSK9 level and risk of CVDs. It is clear that PCSK9 level in the plasma is an independent risk factor beyond the traditional cardiovascular risk factors and indicates a potential role of PCSK9 in the risk assessment. ${ }^{4,5}$ However, earlier studies by Ridker et al and others were not consistent with these findings, which may be due to circadian variations in the synthesis of PCSK9 in the liver, which may be under influence of circadian clock. ${ }^{17-19}$ High levels of PCSK9 can predispose atherosclerosis as shown in Figure 1

The Brisighella Heart Study, selected 1296 subjects including; 227 premenopausal women and 193 age-matched men and 460 postmenopausal women and 416 age-matched men. ${ }^{20}$ The results showed that postmenopausal women had higher PCSK9 levels (309.9 $\pm 84.1 \mathrm{ng} / \mathrm{mL})$ compared with the other groups $(\mathrm{P}<0.001)$. The mean level of PCSK9 was significantly higher in the older men than younger men $(283.2 \pm 75.6$ versus $260.9 \pm 80.4 \mathrm{ng} / \mathrm{mL}$; $\mathrm{P}=0.008)$. In the whole sample, pulse wave velocity was predicted mainly by age $(\mathrm{B}=0.116,95 \%$ CI $0.96-0.127, \mathrm{P}<0.001)$ and levels of PCSK9 $(\mathrm{B}=0.014,95 \%$ CI $0.011-0.016, \mathrm{P}<0.001)$, and serum uric acid $(\mathrm{B}=0.313,95 \%$ CI $0.024-0.391, \mathrm{P}=0.026)$. It is clear that circulating PCSK 9 is significantly related to arterial stiffness, independent of sex and menopausal status in women. ${ }^{20}$ The increased risk of diabetes among subjects receiving statins could be due to beta cell damage caused by lower cell membrane cholesterol and high PCSK9 levels. ${ }^{21}$

It seems that there are other mechanisms by which increase in PCSK9 increases serum levels of LDL cholesterol (LDL receptor degradation), increases intestinal triglyceride-rich lipoprotein production and secretion through transcriptional and posttranscriptional mechanisms. ${ }^{22-24}$ Increased PCSK9 also enhances triglyceride accumulation by targeting the very LDL receptor in the adipose tissue..$^{25,26}$ There is experimental evidence that PCSK9 may be significantly expressed in vascular smooth muscle cells as well as in the human atherosclerotic plaques. ${ }^{27}$ Further evidence revealed that circulating PCSK9 may also be involved in neointima formation. ${ }^{28}$ There is an increase in total plaque area with increase in PCSK9(100ng/mL increase). ${ }^{29}$ In familial hypercholesterolemic and hypertensive patients, PCSK9 levels were associated with carotid intima-media thickness. ${ }^{30,31}$ In view of these adverse effects of PCSK9 in the cardiovascular tissues, PCSK9 inhibiters have been used for decline in CVDs with some success. ${ }^{9,32}$ In a prospective study, 181 patients presenting with ST-segment-elevation myocardial infarction, undergoing primary percutaneous coronary intervention, were prospectively enrolled with a follow-up of 24 months. ${ }^{33}$ Eosinophil cationic protein serum levels predicted major adverse cardiac events after follow up of 2 years (odds ratio $=1.041,95 \%$ confidence interval 1.012-1.071, $\mathrm{P}=0.005$ ), although $\mathrm{C}$-reactive protein serum levels showed only a borderline statistical significance. It is possible that increased eosinophil count could be an indicator of inflammation (allergic) which may be possibly due to increase in PCSK9 levels, which is associated with worst clinical outcome in ACS.

\section{PCSK9 inhibiters and cardiovascular diseases}

Since PCSK9 is a protein secreted from PCSK9 gene, human monoclonal antibodies are used for the reduction of PCSK9 concentrations. A recent meta-analysis including 35 randomized controlled trials comprising 45539 patients, mean age was $61.0 \pm 2.8$ years, had a mean follow-up: 85.5 weeks. ${ }^{9}$ Treatment with a PCSK9 inhibitor was associated with a lower rate of myocardial infarction (2.3\% versus 3.6\%; odds ratio [OR]: 0.72 [95\% confidence interval (CI), 0.64-0.81]; $P<0.001)$, stroke $(1.0 \%$ versus $1.4 \%$; OR: 0.80 [95\% CI, $0.67-0.96] ; P=0.02)$, and coronary revascularization (4.2\% versus 5.8\%; OR: 0.78 [95\% CI, $0.71-0.86] ; P<0.001$ ) compared 
to control group. However, no significant reduction was reported in all-cause mortality (OR: 0.71 [95\% CI, 0.47-1.09]; $P=0.12$ ) or cardiovascular mortality (OR: 1.01 [95\% CI, 0.85-1.19]; $P=0.95$ ). A significant association was observed between higher baseline LDL cholesterol and benefit in all-cause mortality $(P=0.038)$. It is clear that administration of PCSK9 inhibitor is well tolerated and improves CVDs, without any overall benefit in all-cause or cardiovascular mortality. ${ }^{9}$ But, such benefit may be possible in patients with higher baseline LDL cholesterol, hence larger trials are necessary to confirm that reduction in PCSK9 can cause significant reduction in CAD and CVD mortality. The ODYSSEY OUTCOMES trial comprising of 18 924 patients of ACS from 57 countries were followed up for 4years. In patients with recent ACS, alirocumab 75 or $150 \mathrm{mg}$ subcutaneous Q2W targeting LDL-C levels $25-50 \mathrm{mg} / \mathrm{dl}$ and allowing levels as low as $15 \mathrm{mg} / \mathrm{dL}$ compared to placebo, reduced major cardiovascular events, myocardial infarction and ischemic stroke (Table 1). Treatment with alirocumab was associated with a significantly lower rate of all-cause death and was safe and well-tolerated over the duration of the trial.

Table I Main secondary efficacy endpoints: Hierarchical testing CAD, coronary artery disease, $\mathrm{MI}$, myocardial infarction

\begin{tabular}{|c|c|c|c|c|}
\hline End points, $n(\%)$ & Alirocumab( $n=9462)$ & Placebo(n=9462) & $\begin{array}{l}\text { HR(95\% } \\
\text { interval) }\end{array}$ & $\begin{array}{l}\text { Long- rank } P \\
\text { value }\end{array}$ \\
\hline CAD events & $1199(12.7)$ & $1199(12.7)$ & $0.88(0.81,0.95)$ & 0.001 \\
\hline Major CAD events & $793(8.4)$ & $899(9.5)$ & $0.88(0.80,0.96)$ & 0.006 \\
\hline Cardiovascular events & $130 \mid(\mid 3.7)$ & $1474(\mid 5.6)$ & $0.87(0.81,0.94)$ & 0.0003 \\
\hline Death, $\mathrm{MI}$, ischemic stroke & $973(10.3)$ & II26 (II.9) & $0.86(0.79,0.93)$ & 0.0003 \\
\hline CAD Death & $205(2.2)$ & $222(2.3)$ & $0.92(0.76, I . I I)$ & 0.38 \\
\hline Cardiovascular death & $240(2.5)$ & $27 I(2.9)$ & $0.88(0.74,1.05)$ & 0.15 \\
\hline All cause death & $334(3.5)$ & $392(4.1)$ & $0.85(0.73,0.98)$ & 0.026 \\
\hline
\end{tabular}

It is clear that in this nearly 19,000-patient placebo-controlled trial, including many patients treated for $\geq 3$ years, there was no safety signal with alirocumab other than injection site reactions. Among patients with ACS and baseline LDL cholesterol $\geq 100 \mathrm{mg} /$ $\mathrm{dL}$, alirocumab reduced MACE by $24 \%$ (ARR 3.4\%) and all-cause death by $29 \%$ (ARR $1.7 \%$ ) compared with placebo. This trial provides a proof that alirocumab did not cause a significant decline in CAD and $\mathrm{CV}$ mortality. It is clear that new strategies are necessary to improve the efficacy of alirocumab while manipulating its dose and mode of administration without causing any adverse effects. It may be possible via using Franz Halberg's approach by finding out the chrono-pharmacokinetics of alirocumab and chrono-bio kinetics of LDL receptor activity and PCSK9 release. ${ }^{35-38}$

\section{Circadian regulation of PCSK9 expression}

There is some evidence that the synthesis of LDL cholesterol and circulating PCSK9 concentrations are circadian periodic, which gives us an opportunity to use Halberg's approach of chronotherapy with monoclonal antibodies, to enhance their efficacy and reduce adverse effects of these agents. ${ }^{12,35-38}$ It has been demonstrated long ago that disruption of the body clock may be a risk factor for metabolic syndrome. ${ }^{35-40}$ However, there is a need to find out how the circadian pacemaker interacts with the genetic factors associated with plasma lipid traits. Recent genome-wide association studies have identified an expanding list of genetic variants that influence plasma cholesterol and triglyceride levels. ${ }^{39} \mathrm{~A}$ recent analyzed circadian regulation of lipid-associated candidate genes in the liver and identified two distinct groups exhibiting rhythmic and non-rhythmic patterns of expression during light-dark cycles. The disruption of the PCSK9/LDL receptor regulatory axis via liver-specific inactivation of Bmall was associated with increase in plasma LDL/VLDL cholesterol levels. It could be a consequence of ablation of the liver clock perturbed diurnal regulation of lipid-associated genes in the liver and markedly reducing the expression of the non-rhythmically expressed gene Trib $1 .{ }^{39}$ The experiment reported that adenovirus-mediated rescue of Trib1 expression lowered plasma PCSK9 levels, increased LDL receptor protein expression, and restored plasma cholesterol homeostasis, in absence of a functional liver clock. This mechanism appears to be unexpected, regulated by circadian clock through which it controls cholesterol homeostasis by regulation of non-rhythmic genes in the liver (Figure 2).

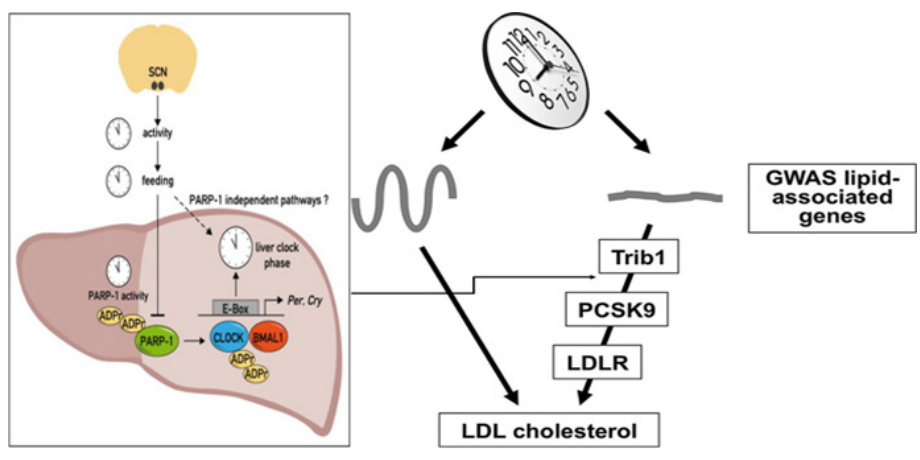

Figure 2 Showing liver clock regulating trib I non-rhythmic genes that acts on PCSK9/LDL receptor axis to regulate plasma cholesterol. ${ }^{39}$

Any alteration in the concentration of PCSK9 in the plasma may be synchronous with synthesis of cholesterol by the hepatocytes under conditions of no therapy to reduce cholesterol. Further studies indicate that disruption of the circadian rhythm of the gene in hepatocytes in its expression in an specific aryl hydrocarbon receptor nuclear translocator-like protein $1(=$ Bmal1) can cause marked rise in PCSK9 concentrations with a decline in LDL receptor and increase in LDL cholesterol levels. ${ }^{39}$ The targets of the Bmall gene are hundreds of other genes via regulation of transcription. Other genes may include all of the clock genes and a number of genes responsible for regulation of encoding metabolism. The expression of the Tribbles homolog 1, may be decreased due to loss of Bmal1. Tribs1 is a protein responsible for the turnover of transcription factor CCAAT/enhancerbinding protein $\alpha(\mathrm{C} / \mathrm{EBP} \alpha)$. In the activation of mitogen activated protein (MAP) kinase and phosphorylation can result in the inhibition of transcription of lipogenic gene including PCSK9 gene. ${ }^{39,40}$ The variants near the TRIB1 gene locus and all the traits of plasma lipids have repeatedly been correlated genome-wide. ${ }^{41}$ The PCSK9 transcription may be induced by peroxisome proliferator-activated receptor $\gamma(\operatorname{PPAR} \gamma)$ which may activate MAP kinase activation and 
may reduce the expression of PCSK9 via dephosphorylation of PPAR $\gamma$. It is possible that inhibition of MAP kinase induces PCSK9 expression in the hepatocytes (HepG2 cells). ${ }^{42}$ There is increased processing of SREBP-2 due to the effects of PPAR $\gamma$ activity on transcription of PCSK 9.42

It is important to gain insight into the circadian function of PCSK9 in humans by establishing whether circulating levels are influenced by diurnal, dietary, and hormonal changes. ${ }^{12}$ In a clinical study, circulating PCSK9 were measured, in a set of dynamic human experiments which show that serum PCSK9 levels display a diurnal rhythm that closely parallels that of cholesterol synthesis, measured as serum lathosterol. Despite marked diurnal changes in cholesterol metabolism, serum LDL cholesterol levels remained stable during the diurnal cycle. However, decline in liver cholesterol by treatment with the bile acid-binding resin, cholestyramine, abolished the diurnal rhythms of both PCSK9 and lathosterol. The intervention with fasting (>18hours) was associated with reduction in circulating PCSK9 and lathosterol levels, whereas serum LDL levels remained unchanged. The increase in growth hormone, during fasting in humans, was associated with reduction in circulating PCSK9 in parallel to LDL cholesterol levels. There was a marked variation in circulating PCSK9 levels, throughout the day, and in response to fasting and cholesterol depletion possibly, due to stimulation of circadian clock which regulates the metabolism of hepatocytes according to time structures. ${ }^{12}$ It is possible that these changes may be due to oscillations in hepatic cholesterol that modify its activity in parallel with cholesterol synthesis. It is also possible that this sterol-mediated regulation, may have additional effects on LDL receptors that may be mediated by hormones directly influencing PCSK9. Apart from its effects on LDL receptor activity, PCSK9 concentration may have circadian stage effects on pro-inflammatory cytokines and endothelial function which may also be circadian periodic. The concentration of PCSK9 can also be altered by nutraceuticals without any adverse effects. ${ }^{16}$ This study demonstrated that cardiovascular risk can be reduced by improving lipid-related serum lipoprotein functions such as high-density lipoproteins cholesterol efflux capacity and serum cholesterol loading capacity apart from reducing PCSK9, independently of lipoprotein concentrations.

\section{Chronotherapy with pcsk9 inhibitors}

Similar to LDL receptor activity, PCSK9 concentrations may follow a circadian variation synchronized with cholesterol synthesis, indicating that the bioactivity of PCSK9 in the body may have circadian stage influence on its beneficial and adverse effects. It is possible that PCSK9 inhibitors may also provide circadian periodic effects which need further characterization since a molecule could be highly bio available but may not be bioactive. ${ }^{35-38}$

In a recent view point, Singh et al, advised to assess the circadian variation in PCSK9 levels by cosinor using Halberg,s approach. ${ }^{38}$ The PCSK9 concentrations in the blood should be determined every 4hours for 7- days in order to find the time (circadian stage) of its highest activity, so that treatment with evolocumab or alirocumab or any other agent, may be optimized by timing in relation to each patient's time of awakening. Chronotherapy takes into consideration the fact that physiology and metabolism follow a 24 hour synchronized rhythm, coordinated by a central circadian clock present in the suprachiasmatic nucleus (SCN) as well as in peripheral clocks present in all other organs, such as hepatocytes in the liver. A chrono therapeutic approach can thus result in increased efficacy and decreased toxicity, even with lower doses of the agent as compared to conventional drug administration (without consideration for timing). Chronotherapy with evolocumab and alirocumab is expected to enhance its bioactivity and to be associated with a decrease in deaths due to CAD and stroke observed in the recent clinical trials, and in its other adverse effects in patients with ACS. ${ }^{9,18,34}$ Chronotherapy with PCSK9 inhibitors will have the ethical advantage of reducing drug consumption and toxicity while increasing efficacy by influencing circadian clock in the brain and liver.

In brief, Apart from LDL receptor degradation, increase in PCSK9 concentrations can also have adverse effects on inflammation, endothelial dysfunction and hypertension independent of their effects on LDL receptors. Since PCSK9 levels and LDL receptor activity are circadian periodic, causing increased synthesis and turnover of cholesterol in the night, there is a need to find out circadian concentration of PCSK9 in the plasma according to time structure. In view of the significant adverse effects and usefulness of PCSK9 inhibitors (monoclonal antibodies), there is a need for new clinical trials via chronotherapy with PCSK9 inhibitors by using circadian stage PCSK9 concentration in patients with ACS. This is important because deaths due to CAD and stroke have become worldwide public health problems which may be prevented by chronotheraphy.

\section{Acknowledgements}

Acknowledgements are due to International College of Cardiology for logistic support to write this article.

\section{Conflict of interest}

Conflict of interest has not been declared by the authors.

\section{References}

1. Lakoski SG, Lagace TA, Cohen JC, et al. Genetic and metabolic determinants of plasma PCSK9 levels. J Clin Endocrinol Metab. 2009;94(7):2537-2543.

2. Shapiro MD, Fazio S. PCSK9 and atherosclerosis-lipids and beyond. $J$ Atherosclerosis Thrombosis. 2017;24(5):462-472.

3. Lambert G, Charlton F, Rye KA, et al. Molecular basis of PCSK9 function. Atherosclerosis. 2009;203(1):1-7.

4. Qiu C, Zhou Q, Li X, et al. High circulating proprotein convertase subtilisin/Kexin type 9 concentration associates with cardiovascular risk:A meta-analysis of cohort studies. Medicine (Baltimore). 2017;96(48):e8848.

5. Xiao Y, Peng C, Huang W, et al. Circulating proprotein convertase subtilisin/ Kexin type 9 (PCSK9) concentration and risk of cardiovascular eventssystematic review and meta-analysis of prospective studies. Circulation. 2017;81(8):1150-1157

6. Fedacko J, Singh RB, Gaspar L, et al. Efficacy and safety outcomes in clinical trials of proprotein convertase subtilisin-kexin (PCSK) type 9 inhibitors in coronary artery disease. World Heart J. 2017;9(3).

7. Singh RB, Hristova K, Fedacko A, et al. Recent monoclonal antibody trials in the management of coronary artery disease. World Heart J. 2015;7:8992

8. Vargova V, Singh RB, Gaspar L, et al. Recent clinical trials of proprotein convertase subtilisin-kexin (PCSK) type 9 inhibitors in coronary artery disease. World Heart J. 2017;9:15-18

9. Karatasakis A, Danek BA, Karacsonyi J, et al. Effect of PCSK9 inhibitors on clinical outcomes in patients with hypercholesterolemia:A meta-analysis of 35 Randomized Controlled Trials. J Am Heart Assoc. 2017;6(12):e006910. 
10. Singh P, Singh RB, Fedacko J, et al. Proprotein convertase subtilisin kexin 9 (PCSK9) and lipoprotein metabolism in relation to adverse birth outcome and future cardiometabolic risk. World Heart J. 2016;8:95-99.

11. Fedacko J, Hristova K, Elkilany G, et al. Can chronotherapy enhance efficacy and diminish adverse effects of PCSK9 inhibitors in the management of acute coronary syndromes? World Heart J. 2015;7:97-102.

12. Persson, L, Cao G, Stahle L, et al. Circulating proprotein convertase subtilisin kexin type 9 has a diurnal rhythm synchronous with cholesterol synthesis and is reduced by fasting in humans. Arterioscler Thromb Vasc Biol. 2010;30(12):2666-2672.

13. Glerup S, Schulz R, Laufs U, et al. Physiological and therapeutic regulation of PCSK9 activity in cardiovascular disease. Basic Research in Cardiology. 2017;112(3):32.

14. Dubuc G, Tremblay M, Paré G, et al. A new method for measurement of total plasma PCSK9:clinical applications. Journal of Lipid Research. 2010;51(1):140-149.

15. Urban D, Pöss J, Böhm M, et al. Targeting the proprotein convertase subtilisin/kexin type 9 for the treatment of dyslipidemia and atherosclerosis J Am Coll Cardiol. 2013;62(16):1401-1408.

16. Adorni MP, Ferri N, Marchianò S, et al. Effect of a novel nutraceutica combination on serum lipoprotein functional profile and circulating PCSK9. Ther Clin Risk Manag. 2017;13:1555-1562.

17. Ridker PM, Rifai N, Bradwin G, et al. Plasma proprotein convertase subtilisin/kexin type 9 levels and the risk of first cardiovascular events. Eur Heart J. 2016;37(6):554-560.

18. Fedacko J, Moshiri M, Singh RB, et al. Beyond cholesterol: the proprotein convertase subtilisin/kexin type 9 that increases the risk of atherosclerosis. World Heart J. 2018;10:17-24.

19. Ma D, Liu T, Chang L, et al. The liver clock controls cholesterol homeostasis through Trib1 protein-mediated regulation of PCSK9/low density lipoprotein receptor (LDL-R) axis. J Biol Chem. 2015;290(52):3100331012.

20. Ruscica M, Ferri N, Fogacci F, et al. Circulating levels of proprotein convertase subtilisin/kexin type 9 and arterial stiffness in a large population sample: data from the brisighella heart study. J Am Heart Assoc. 2017;6(5):e005764.

21. Verma NS, Singh RB, Saboo B, et al. Proprotein convertase subtilisinkexin (PCSK9) type 9 inhibitors with reference to risk of diabetes. World Heart J. 2017;10:9-14.

22. Ferri N, Corsini A, Macchi C, et al. Proprotein convertase Subtilisin kexin type 9 and high-density lipoprotein metabolism:experimental anima models and clinical evidence. Transl Res. 2016;173:19-29.

23. Lambert G, Sjouke B, Choque B, et al. The PCSK9 decade. J Lipid Res 2012;53(12):2515-2524

24. Norata GD, Tavori H, Pirillo A, et al. Biology of proprotein convertase subtilisinkexin 9: beyond low-density lipoprotein cholesterol lowering. Cardiovasc Res. 2016;112(1):429-442.

25. Roubtsova A, Munkonda MN, Awan Z, et al. Circulating proprotein convertase subtilisin/ kexin 9 (PCSK9) regulates VLDLR protein and triglyceride accumulationin visceral adipose tissue. Arterioscler Thromb
Vasc Biol. 2011;31(4):785-791.

26. Ferri N, Ruscica M. Proprotein convertase subtilisin/kexin type 9 (PCSK9) and metabolic syndrome: insights on insulin resistance, inflammation, and atherogenic dyslipidemia. Endocrine. 2016;54(3):588-601.

27. Ferri N, Tibolla G, Pirillo A, et al. Proprotein convertase subtilisinkexin type 9 (PCSK9) secreted by cultured smooth muscle cells reduces macrophages LDLR levels. Atherosclerosis. 2012;220(2):381-386.

28. Ferri N, Marchian OS, Tibolla G, et al. PCSK9 knock-out mice are protected from neointimal formation in response to perivascular carotid collar placement. Atherosclerosis. 2016;253:214-224

29. Xie W, Liu J, Wang W, et al. Association between plasma PCSK9 levels and 10-year progression of carotid atherosclerosis beyond LDL-C: a cohort study. Int J Cardiol. 2016;215:293-298.

30. Huijgen R, Fouchier SW, Denoun M, et al. Plasma levels of PCSK9 and phenotypic variability in familial hypercholesterolemia. J Lipid Res. 2012;53(3):979-983.

31. Lee CJ, Lee YH, Park SW, et al. Association of serum proprotein convertase subtilisin/kexin type 9 with carotid intima media thickness in hypertensive subjects. Metabolism. 2013;62(6):845-850.

32. Saboo B, Singh RB, Fedacko J, et al. Proprotein convertase subtilisin/kexin type 9 (PCSK9) inhibitors. World Heart J. 2015;7:89-96.

33. Niccoli G, Calvieri C, Flego D, et al. Allergic inflammation is associated with coronary instability and a worse clinical outcome after acute myocardial infarction. Circ CardiovascInterv. 2015;8(8):e002554.

34. Schwartz GG, Szarek M, Bhatt DL, et al. On behalf of the ODYSSEY OUTCOMES Investigators and Committees American College of Cardiology-The ODYSSEY OUTCOMES Trial: Topline Results Alirocumab in Patients After Acute Coronary Syndrome. 2018

35. Halberg F, Cornelissen G, Wang ZR, et al. Chronomics: circadian and circaseptan timing of radiotherapy, drugs, calories, perhaps nutriceuticals and beyond. J Exp Therapeutics Oncol. 2003;3(5):223-260.

36. Singh RB, Darlenska TS, Hristova K, et al. Chronocardiology and chronotherapy. J Cardiol Ther. 2014;2(1):31-36.

37. Cornelissen G. Cosinor-based rhythmometry. Theoretical Biology and Medical Modelling. 2014;11:16.

38. Singh RB, Cornelissen G, Gvozdjakova A, et al. Can Halberg's chronotherapy approach increase efficacy and safety of PCSK9 inhibitors and decrease their adverse effects? WHJ. 2015;7:93-96.

39. Ma D, Liu T, Chang L, et al. The liver clock controls cholesterol homeostasis through Trib1 protein-mediated regulation of PCSK9/low density lipoprotein receptor (LDL-R) axis. J Biol Chem. 2015;290:31003-31012.

40. Bauer RC, Yenilmez BO, Rader DJ. Tribbles-1: a novel regulator of hepatic lipid metabolism in humans. Biochem Soc Trans. 2015;43(5):1079-1084.

41. Willer CJ, Schmidt EM, Sengupta S, et al. Discovery and refinement of loci associated with lipid levels. Nat Genet. 2013;45(11):1274-1283.

42. Duan Y, Chen Y, Hu W, et al. Peroxisome proliferator-activated receptor gamma activation by ligands and dephosphorylation induces proprotein convertase subtilisin kexin type 9 and low density lipoprotein receptor expression. J Biol Chem. 2012;287(28):23667-23677. 